\title{
Physical and Metallurgical Views on the Process of Chip Creation
}

Karol Vasilko

Faculty of Manufacturing Technologies, Technical University in Košice with the seat in Prešov, 08001 Prešov, Bayerova 1, SR, karol.vasilko@tuke.sk

To understand the process of chip creation during machining requires knowing the behaviour of metal at high degrees of mechanical and thermal stress which occurs in microvolumes of machined material. During machining the chip is characterized by unique deformation structure which does not occur with other methods of mechanical metal machining. This is the reason why its explanation is rather complex. The implementation of the knowledge of metal physics in confrontation with the experiment can finally contribute to the optimization of the process of metal machining and the selection of optimal cutting conditions.

Keywords: cutting, chip formation, cutting forces, stress

\section{References}

[1] LOLADZE, T. N. (1952). Stružkoobrazovanije pri rezanii metallov. Moskva: Mašgiz 1952.

[2] ŠLESÁR, M. (1967). Mikroprocesy deformácie a porušovania ocelí vo vzt’ahu k vlastnostiam a morfológii koexistujúcich fáz. Kovové materiály, 1967, No. 3, pp. 221-242

[3] ŠALAK, A, SELECKÁ, M., DANNINGER, H. (2005). Machinability of powder Metallurgy Steels. Cambridge international Science Publishing, 2005, p. 836, ISBN 1-898326-82-7

[4] Patent SR, 122243: Nová metóda na zastavenie procesu obrábania bez špeciálnych prípravkov. Pôvodca: BUDA, J., VASILKO, K., 1967.

[5] BUDA, J., BÉKÉS, J. (1977). Teoretické základy obrábania kovov, Bratislava: ALFA, 1977, 698 s.

[6] GRZESIK, W. (2010). Podstawy skrawania materialow metalowych. Warszawa: Wydawnictwa Naukowo-Techniczne, 2010, 526 s., ISBN 978-83-204-3668-6

[7] TRENT, E. M., WRIGHT, P. K. (2000). Metal Cutting. Butterworth Heinemann, 2000, 446 p., ISBN 0-75067069-X

[8] MORIVAKI, T., IWATA, K., NAKAYAMA, K. (2006). Chip Formation Mechanism in Single Crystal Cutting of Beta-Brass. Annals of the CIRP, 55/1, 2006, 103-106

[9] KALLIOPIN, V. V. (1969). Mechanika volny pri rezanii. Nauka i technika, Minsk, 1969

[10] UHLMANN, M. (2007). Finite Element Modelling and Cutting Simulation of Inconel 718. Annals of the CIRP Vol. 56/1/2007, pp. 61-64

[11] CERETTI, E., FILICE, L., UMBRELLO, F., MICAN, F. (2007). ALE Simulation of Orthogonal Cutting: a new Approach to Model Heat Transfer Phenomena at the Tool-Chip Interface. Annals of the CIRP Vol. 56/1/2007, pp.69-72

[12] MURČINKO, J., MURČINKOVÁ, Z. (2009). Monitoring of vibrations dangerous limits in machining process of BÖHLER M 201 material In: Proceedings in Manufacturing Systems. Vol. 4 (2009), p. 217-220. - ISSN 18423183.

[13] REZNIKOV, A.N. (1969). Teplofizika rezanija. Moskva: Mašinostrojenije, 1969, 286s.

[14] SMART, E. F, TRENT, E. M. (1985). Distribution des temperatures dans les outils de couple utilizes pour 1' usinage du far, du titane et du nickel. Bull. Cerlce étud. Métaux, 1985, num. spac., 443-447. Discuss, 478-479.

[15] WRIGHT, P. K. (1977). Applications of the Experimental Methods Used to Determine Temperature Gradients. In: Cutting Tools. Austrian Conference Manufacturing Engineering., Adelaide, 1977. Barton, 1977, pp. 145-149

[16] VASILKO, K., MÁDL, J. (2012). Teorie obrábění. Ústí nad Labem: 2012, 2015, 526 s., ISBN 978-80-7414-4608

[17] MOORE, D F. (1975). Principles and Applications of Tribology. ASME 1975

[18] DEGNER, W. (1968). Beitrag zur Untersuchung von Oberflächenbeschaffenheit des Werkstückes Wissenschaftliche Zeitschrift Technisch Hochschule Karl Marx Stadt, 1968, 10, s. 53-61. 
[19] SYREN, B., WOHLFAHRT, H., MACHERAUCH, E. (1977). Zur Emtstehung von Bearbeitungseigenspannungen. Arch. Eisenhüttenw., 1977, 48, Nr.8, 421-426

[20] ZOREV, N. N. (1963). O vzaimozavisivosti processov v zone stružkoobrazovanija I v zone kntakta perednej poverchnosti instrumenta. Vestnik mašinostrjenija, 1963, No. 12.

[21] LOLADZE, T. N. (1982). Pročnost’ i iznosostojkost’ režuščego instumenta. Moskva: Mašinostrojenije, 1982,318 p.

[22] BACH,P., POLÁČEK, M., CHVOJKA, P., DROBÍLEK, J. (2014). Dynamic Forces in Unstable Cutting during Turning Operation. Manufacturing Technology, 2014, Vol 14, No.1, pp. 3-8, ISSN 1213-2489

[23] DUGIN, A., POPOV, A. (2012). Effect of the processing materials on the ploughing force values. Manufacturing Technology, 2012, vol. 12, No. 13, pp. 102-105

[24] PÁLMAI, Z. (2012). Model of chip formation during turning in the presence of a built-up edge (BUE). Manufacturing Technology, 2012, Vol.12, No.13, pp. 207-212, ISSN 1213-2489

[25] KRÓLCZYK G., LEGUTKO S. (2013). The machinability of duplex stainless steel - solutions in practice, Manufacturing Technology, 2013, 13 (4), pp. 473 - 478

[26] KRÓLCZYK G., NIESŁONY P., LEGUTKO S., STOIC A. (2014). Microhardness changes gradient of the duplex stainless steel (DSS) surface layer after dry turning, Metalurgija 2014, 53/4, 529-532. 